\title{
Development of an Open-Heart Intraoperative Risk Scoring Model for Predicting a Prolonged Intensive Care Unit Stay
}

\author{
Sirirat Tribuddharat, ${ }^{1}$ Thepakorn Sathitkarnmanee, ${ }^{1}$ \\ Kriangsak Ngamsangsirisup, ${ }^{1}$ Somrat Charuluxananan, ${ }^{2}$ Cameron P. Hurst, ${ }^{3}$ \\ Suparit Silarat, ${ }^{1}$ and Ganjana Lertmemongkolchai ${ }^{4}$ \\ ${ }^{1}$ Department of Anesthesiology, Faculty of Medicine, Khon Kaen University, Khon Kaen 40002, Thailand \\ ${ }^{2}$ Department of Anesthesiology, Faculty of Medicine, Chulalongkorn University, Bangkok 10330, Thailand \\ ${ }^{3}$ Clinical Epidemiology Unit (CEU), Faculty of Medicine, Khon Kaen University, Khon Kaen 40002, Thailand \\ ${ }^{4}$ Department of Clinical Immunology, Faculty of Associated Medical Sciences, Khon Kaen University, Khon Kaen 40002, Thailand
}

Correspondence should be addressed to Thepakorn Sathitkarnmanee; thepakorns@gmail.com

Received 6 February 2014; Revised 7 March 2014; Accepted 21 March 2014; Published 10 April 2014

Academic Editor: Cheng I. Cheng

Copyright (C) 2014 Sirirat Tribuddharat et al. This is an open access article distributed under the Creative Commons Attribution License, which permits unrestricted use, distribution, and reproduction in any medium, provided the original work is properly cited.

Background. Based on a pilot study with 34 patients, applying the modified sequential organ failure assessment (SOFA) score intraoperatively could predict a prolonged ICU stay, albeit with only 4 risk factors. Our objective was to develop a practicable intraoperative model for predicting prolonged ICU stay which included more relevant risk factors. Methods. An extensive literature review identified 6 other intraoperative risk factors affecting prolonged ICU stay. Another 168 patients were then recruited for whom all 10 risk factors were extracted and analyzed by logistic regression to form the new prognostic model. Results. The multivariate logistic regression analysis retained only 6 significant risk factors in the model: age $\geq 60$ years, $\mathrm{PaO}_{2} / \mathrm{FiO}_{2}$ ratio $\leq 200 \mathrm{mmHg}$, platelet count $\leq 120,000 / \mathrm{mm}^{3}$, requirement for inotrope/vasopressor $\geq 2$ drugs, serum potassium $\leq 3.2 \mathrm{mEq} / \mathrm{L}$, and atrial fibrillation grading $\geq 2$. This model was then simplified into the Open-Heart Intraoperative Risk (OHIR) score, comprising the same 6 risk factors for a total score of 7-a score of $\geq 3$ indicating a likely prolonged ICU stay (AUC for ROC of 0.746). Conclusions. We developed a new, easy to calculate OHIR scoring system for predicting prolonged ICU stay as early as 3 hours after CPB. It comprises 6 risk factors, 5 of which can be manipulated intraoperatively.

\section{Introduction}

Cardiac surgery with cardiopulmonary bypass $(\mathrm{CPB})$ is associated with immune responses harmful to the patient, often called "post-bypass syndrome" $[1,2]$. It may contribute to life-threatening perioperative complications and even multiorgan dysfunction syndrome (MODS) or multiorgan failure (MOF), which causes considerable clinical morbidity, mortality, and eventually a prolonged stay in the intensive care unit (ICU)/hospital [3, 4]. Early evaluation of the severity of the intraoperative immunoinflammatory response, with a proper predictive model, is helpful for (a) recognizing the possible increase in risk, (b) planning proper management, (c) reducing postoperative complications, (d) improving outcomes, and (e) decreasing the length of stay in intensive care (ICU) and hospital.

There are several predictive models for prolonged ICU stay, but all are used preoperatively for proper resource management [5]. What is lacking, therefore, is a predictive model for intraoperative use, which identifies correctable risk factors that can be addressed for improving patient care and prognosis.

Prognostic scoring systems for assessing the severity of inflammation and predicting the mortality rate and length 
of ICU stay include (a) the acute physiology and chronic health evaluation (APACHE) II; (b) the sequential organ failure assessment (SOFA); and (c) the multiorgan dysfunction (MOD) scores [6]. SOFA is the simplest to calculate at bedside and may be used to predict prolonged stay in ICU after cardiac surgery. We conducted a pilot study among 34 patients undergoing cardiac surgery with $\mathrm{CPB}$ to assess the ability of a modified SOFA score for predicting prolonged ICU stay. We, however, excluded the Glasgow coma score because such patients were unconscious from general anesthesia. The modified SOFA 3 hours after CPB initiation predicted a prolonged ICU stay but was not suitable for intraoperative assessment because it comprises only 4 known relevant risk factors, namely, $\mathrm{PaO}_{2} / \mathrm{FiO}_{2}(\mathrm{P} / \mathrm{F})$ ratio, inotropic/vasopressor drugs used, platelet count, and creatinine level. A new intraoperative prognostic model with more relevant correctable risk factors is needed.

Our objective was to develop a new, simple, prognostic model to be used intraoperatively to predict prolonged ICU stay and as a tool for improving patient care in cardiac surgery.

\section{Methods}

This was a prospective, observational, exploratory, analytical study. The experimental protocol was approved by the Human Research Ethics Committee, Khon Kaen University (HE531033 and HE541122), and registered at ClinicalTrials.gov (NCT01353157 and NCT01559870). The pilot study was conducted between February and June 2010 and the model development between July and December 2011 at Queen Sirikit Heart Center of the Northeast, Khon Kaen University, Thailand. All of the patients gave written, informed consent before recruitment.

An extensive literature review revealed another 6 relevant risk factors influencing prolonged ICU stay [7-18], namely, age, New York Heart Association (NYHA) classification, ejection fraction, hematocrit level, serum potassium level, and atrial fibrillation grading. According to Tabachnick and Fidell [19], the required sample size $(N)$ for a logistic regression of a full model should ideally be $50+10(k)$ : for us this meant 168 patients based on 10 relevant clinical risk factors $(k)$ and a dropout margin of $10 \%$.

We included any patient, between 18 and 75 years of age, with acquired heart disease (including coronary artery disease or valvular heart disease), scheduled for elective cardiac surgery with $\mathrm{CPB}$. We excluded any patient with congenital heart disease and those requiring emergency or redo surgery. Standard anesthetic and surgical techniques were employed for the open-heart surgery with CPB. The anesthesiologists and surgeons were not apprised of the risk factors being studied. A transfusion was indicated in case of (a) hemoglobin level $<8 \mathrm{~g} / \mathrm{dL}$, (b) platelet number $<50,000 / \mathrm{mm}^{3}$, or (c) clinical coagulopathy. The target of fluid management was to maintain a central venous pressure of 8 to $12 \mathrm{mmHg}$ or pulmonary arterial pressure of 12 to $15 \mathrm{mmHg}$ by infusion of crystalloid and colloids. As per protocol, catecholamine infusions were used to support hemodynamic stability, and the sequence of their use was dobutamine then epinephrine or norepinephrine.

After surgery, all of the patients were transferred to the ICU and managed as follows. Before extubation, ventilated patients must (a) be awake and (b) have satisfactory oxygenation and ventilation (i.e., on $\mathrm{FiO}_{2} \leq 40 \%$ with $\mathrm{PaO}_{2}>$ $60 \mathrm{mmHg}, \mathrm{PaCO}_{2}>30$ and $<50 \mathrm{mmHg}$, and $\mathrm{pH}>7.30$, or $\mathrm{SpO}_{2}>92 \%$ ) and (c) be hemodynamically stable. An attending surgeon discharged each patient from the ICU to the cardiovascular ward according to the following criteria: the patient must (a) be alert and cooperative, (b) have a respiratory rate $<25 /$ minute without assistance from mechanical ventilation, (c) have a $\mathrm{PaO}_{2}>80 \mathrm{mmHg}$ and a $\mathrm{PaCO}_{2}<$ $45 \mathrm{mmHg}$, (d) be hemodynamically stable, and (e) have adequate analgesia.

All 10 risk factors were evaluated 3 hours after CPB. Perioperative data were collected, including clinical outcomes and complications. Prolonged ICU stay was defined as an ICU stay of longer than the 42-hour median time in our hospital. All data were extracted for analyses by 2 physicians. The data were checked and any entry errors corrected before the analyses.

2.1. Statistics. The discrimination ability of each predictor was evaluated using the area under the receiver operating characteristic curve (AUC for ROC), for which values $\geq$ 0.600 were considered clinically useful. After determination of the best cut-off points (i.e., by choosing the points with maximum area of the ROC), the continuous values for all of the risk factors were transformed into dichotomous values. Each risk factor was evaluated for the crude odds ratio using the results of the univariate analysis. All of the risk factors with a $P<0.20$ were included in the multivariate logistic regression analysis. Calibration and validation of the model were accomplished using the Hosmer-Lemeshow goodnessof-fit statistic and external validation. The $\alpha$ was set at 0.05. All analyses were performed using GraphPad Prism 5 (GraphPad software, La Jolla, CA, USA) and SPSS version 16.0 for Windows (SPSS, Chicago, IL, USA).

\section{Results}

The demographic and clinical data of 168 patients are presented in Table 1. Table 2 presents the AUC for ROC, crude odds ratios, sensitivity, and specificity for all of the risk factors for predicting prolonged ICU stay. Based on the multivariate logistic regression analysis, only 6 risk factors should be retained in the equation. The regression coefficients and adjusted odds ratios are presented in Table 3. The logistic regression model for predicting the probability of a prolonged ICU stay $(\mathrm{P})$ is 
TABLE 1: Demographic and clinical data.

\begin{tabular}{|c|c|c|c|}
\hline Characteristics & $\begin{array}{c}\text { Nonprolonged ICU } \\
(n=102)\end{array}$ & $\begin{array}{c}\text { Prolonged ICU } \\
\quad(n=66) \\
\end{array}$ & $P$ value \\
\hline Age (years) & $54.5 \pm 11.2$ & $60.5 \pm 12.3$ & 0.002 \\
\hline \multicolumn{4}{|l|}{ Gender } \\
\hline Male & $61(59.8)$ & $54(81.8)$ & \multirow[t]{2}{*}{0.004} \\
\hline Female & $41(40.2)$ & $12(18.2)$ & \\
\hline \multicolumn{4}{|l|}{ Type of operation } \\
\hline CABG & $49(48.0)$ & $36(54.6)$ & 0.433 \\
\hline Valve surgery & $49(48.0)$ & $22(33.3)$ & 0.078 \\
\hline CABG + valve surgery & $4(4.0)$ & $8(12.1)$ & 0.023 \\
\hline \multicolumn{4}{|l|}{ NYHA class } \\
\hline I-II & $83(81.4)$ & $42(63.6)$ & \multirow[t]{2}{*}{0.012} \\
\hline III-IV & $19(18.6)$ & $24(36.4)$ & \\
\hline Ejection fraction (\%) & $59.2 \pm 14.0$ & $53.0 \pm 16.5$ & 0.012 \\
\hline$<40$ & $12(11.8)$ & $16(24.2)$ & 0.055 \\
\hline$\geq 40$ & $90(88.2)$ & $50(75.8)$ & \\
\hline \multicolumn{4}{|l|}{ Preoperative variables: } \\
\hline Hypertension & $33(32.4)$ & $32(48.5)$ & 0.051 \\
\hline Diabetes mellitus & $27(26.5)$ & $22(33.3)$ & 0.386 \\
\hline Myocardial infarction & $16(15.7)$ & $15(22.7)$ & 0.309 \\
\hline Dyslipidemia & $13(12.8)$ & $16(24.2)$ & 0.062 \\
\hline Atrial fibrillation & $20(19.6)$ & $12(18.1)$ & 0.844 \\
\hline Congestive heart failure & $7(6.7)$ & $11(16.7)$ & 0.071 \\
\hline Kidney impairment/disease & $4(3.9)$ & $7(10.6)$ & 0.155 \\
\hline Creatinine value (mg/dL) & $0.95 \pm 0.3$ & $1.11 \pm 0.5$ & 0.025 \\
\hline $\mathrm{CPB}$ time (minutes) & $107.9 \pm 37.9$ & $126.6 \pm 36.9$ & 0.002 \\
\hline Aortic cross-clamping time (minutes) & $78.1 \pm 29.8$ & $89.4 \pm 27.2$ & 0.014 \\
\hline Mechanical ventilation (hours) & $10.9 \pm 7.5$ & $31.8 \pm 41.5$ & $<0.001$ \\
\hline Endotracheal tube retaining (hours) & $11.7 \pm 8.1$ & $33.4 \pm 41.5$ & $<0.001$ \\
\hline ICU stay (hours) & $25.7 \pm 9.3$ & $88.8 \pm 51.6$ & $<0.001$ \\
\hline Hospital stay (days) & $13.6 \pm 5.3$ & $18.5 \pm 9.9$ & $<0.001$ \\
\hline
\end{tabular}

NYHA: New York Heart Association; CABG: coronary artery bypass graft surgery; MVR: mitral valve replacement; MV: mitral valve; AVR: aortic valve replacement; TV: tricuspid valve; CPB: cardiopulmonary bypass; ICU: intensive care unit. Values are mean \pm SD or $n$ (\%).

$$
\begin{aligned}
\mathrm{P}=1 \times(1+\operatorname{EXP}(-(- & .576+0.974 * \text { "Age } \geq 60 \text { years" }+0.857^{*} " \mathrm{P} / \mathrm{F} \text { ratio } \leq 200 \mathrm{mmHg} " \\
& +0.847^{*} \text { "Platelet count } \leq 120 \times 10^{3} / \mathrm{mm}^{3 "} \\
+ & 1.593^{*} \times \text { "Inotrope/vasopressor requirement } \geq 2 \text { drugs" } \\
+ & 0.910^{*} \text { "Serum potassium } \leq 3.2 \mathrm{mEq} / \mathrm{L} " \\
& \left.\left.\left.+0.934^{*} \text { "Atrial fibrillation grading } \geq 2 "\right)\right)\right)^{-1}
\end{aligned}
$$

where $\mathrm{P}=$ probability of a prolonged ICU stay; the values of each risk factor are assigned as follows:

$$
\text { age } \geq 60 \text { years }(\text { yes }=1 \text {; no }=0) \text {; }
$$

$\mathrm{P} / \mathrm{F}$ ratio $\leq 200 \mathrm{mmHg}($ yes $=1 ;$ no $=0)$;

$$
\text { platelet count } \left.\leq 120 \times 10^{3} / \mathrm{mm}^{3} \text { (yes }=1 ; \text { no }=0\right) \text {; }
$$


TABLE 2: Univariate risk factors of a prolonged length of ICU stay.

\begin{tabular}{|c|c|c|c|c|c|c|}
\hline Variable outcomes & AUC for ROC & $95 \% \mathrm{CI}$ & Crude OR & $95 \%$ CI & Sensitivity (\%) & Specificity (\%) \\
\hline \multicolumn{7}{|l|}{ Age (years) } \\
\hline$<60$ & & & 1.0 & & & \\
\hline$\geq 60$ & 0.629 & $0.543-0.716$ & $2.881^{* *}$ & $1.518-5.467$ & 62.1 & 63.7 \\
\hline \multicolumn{7}{|l|}{ NYHA classification } \\
\hline I-II & & & 1.0 & & & \\
\hline III-IV & 0.589 & $0.499-0.678$ & $2.496^{*}$ & $1.231-5.063$ & 36.4 & 81.4 \\
\hline \multicolumn{7}{|l|}{ Ejection fraction (\%) } \\
\hline$>40$ & & & 1.0 & & & \\
\hline$\leq 40$ & 0.562 & $0.472-0.653$ & $2.400^{*}$ & $1.052-5.474$ & 42.4 & 79.4 \\
\hline \multicolumn{7}{|l|}{ Hematocrit (\%) } \\
\hline$>33$ & & & 1.0 & & & \\
\hline$\leq 33$ & 0.570 & $0.483-0.657$ & $3.355^{*}$ & $1.202-9.366$ & 90.9 & 19.6 \\
\hline \multicolumn{7}{|c|}{$\mathrm{PaO}_{2} / \mathrm{FiO}_{2}$ ratio $(\mathrm{mmHg})$} \\
\hline$>200$ & & & 1.0 & & & \\
\hline$\leq 200$ & 0.607 & $0.517-0.696$ & $2.838^{* *}$ & $1.420-5.674$ & 40.9 & 80.4 \\
\hline \multicolumn{7}{|c|}{ Platelet count $\left(/ \mathrm{mm}^{3}\right)$} \\
\hline$>120,000$ & & & 1.0 & & & \\
\hline$\leq 120,000$ & 0.607 & $0.517-0.696$ & $2.838^{* *}$ & $1.420-5.674$ & 40.9 & 80.4 \\
\hline \multicolumn{7}{|c|}{ Inotropic drugs (items) } \\
\hline 1 & & & 1.0 & & & \\
\hline$\geq 2$ & 0.672 & $0.586-0.758$ & $4.932^{* * *}$ & $2.463-9.876$ & 53.0 & 81.4 \\
\hline \multicolumn{7}{|l|}{ Creatinine (mg\%) } \\
\hline$<1.2$ & & & 1.0 & & & \\
\hline$\geq 1.2$ & 0.581 & $0.491-0.671$ & $2.508^{*}$ & $1.192-5.277$ & 31.8 & 84.3 \\
\hline \multicolumn{7}{|c|}{ Potassium level (mEq/L) } \\
\hline$>3.2$ & & & 1.0 & & & \\
\hline$\leq 3.2$ & 0.578 & $0.487-0.668$ & $2.812^{*}$ & $1.251-6.323$ & 27.3 & 88.2 \\
\hline \multicolumn{7}{|c|}{ Atrial fibrillation grading ${ }^{\#}$} \\
\hline$<2$ & & & 1.0 & & & \\
\hline$\geq 2$ & 0.634 & $0.547-0.721$ & $3.020^{* *}$ & $1.588-5.742$ & 59.1 & 67.6 \\
\hline
\end{tabular}

ICU: intensive care unit; AUC for ROC: area under receiver operating characteristic curve; CI: confidence interval; OR: odds ratio; NYHA: New York Heart Association; $\mathrm{PaO}_{2}$ : partial pressure of arterial oxygen; $\mathrm{FiO}_{2}$ : fraction of inspired oxygen in a gas mixture.

${ }^{\#}$ Atrial fibrillation (AF) grading: 0: no AF; 1: AF without tachyarrhythmia and needs no treatment; 2: AF with tachyarrhythmia and needs antiarrhythmic therapy; 3 : AF with tachyarrhythmia and hemodynamic instability and needs antiarrhythmic therapy.

${ }^{*} P<0.05,{ }^{* *} P<0.01,{ }^{* * *} P<0.001$.

inotrope/vasopressor requirement $\geq 2$ drugs (yes $=1$; no $=0$ );

serum potassium $\leq 3.2 \mathrm{mEq} / \mathrm{L}($ yes $=1$; no $=0)$;

atrial fibrillation grading $\geq 2$ (yes $=1$; no $=0$ ).

The AUC for ROC of this model was 0.818 (95\% CI: $0.753-0.883$ ) and, according to the Hosmer and Lemeshow goodness-of-fit test, the model fit the data $(P=0.242)$. Since this model was complex and difficult to calculate, we simplified it into a new Open-Heart Intraoperative Risk (OHIR) score by approximating the adjusted odds ratio around 2.5 as an OHIR score of 1 (Table 4).

The new scoring model includes all 6 risk factors and has a total score of 7 . An OHIR score of $\geq 3$ indicates a prolonged ICU stay (Table 4). The sensitivity, specificity, positive and negative predictive value, overall accuracy, positive likelihood, and AUC for ROC of this model are presented in Table 5. The correlation of the logistic regression and OHIR score models was 0.988 ( $P<0.0001,95 \%$ CI: 0.9837-0.9911) (Figure 1).

Both the logistic regression and OHIR models were externally validated in the 34 patients of the pilot study. The respective AUC for ROC of both models in this validating group was 0.901 (95\% CI: $0.789-1.014)$ and 0.915 (95\% CI: $0.807-1.023)$. The respective sensitivity and specificity of the OHIR score $\geq 3$ for predicting prolonged ICU stay were $81.2 \%$ (95\% CI: 53.7\%-95.0\%) and 94.4\% (95\% CI: 70.6\%-99.7\%).

In order to confirm the validity of applying the OHIR score on our patients, we computed the mean ICU stay for each OHIR score for all of the patients (Table 6). The mean 
TABLE 3: Risk factors associated with a prolonged length of ICU stay from multivariate logistic regression analysis and the OHIR score.

\begin{tabular}{lccc}
\hline Variable outcomes & Regression coefficient & Adjusted OR & $95 \%$ CI \\
\hline Age $(\geq 60$ years $)$ & 0.974 & 2.648 & $1.217-5.762$ \\
P/F ratio $(\leq 200 \mathrm{mmHg})$ & 0.857 & 2.357 & $1.048-5.301$ \\
Platelet count $\left(\leq 120,000 / \mathrm{mm}^{3}\right)$ & 0.847 & 2.333 & $1.011-5.383$ \\
Inotropic drugs $(\geq 2$ items) & 1.593 & 4.920 & 0.014 \\
Potassium level $(\leq 3.2 \mathrm{mEq} / \mathrm{L})$ & 0.910 & 2.485 & 0.047 \\
Atrial fibrillation $($ grading $\geq 2)$ & 0.934 & 2.545 & $0.965-6.394$ \\
Constant & -2.576 & 0.076 & $1.196-5.418$ \\
\hline
\end{tabular}

ICU: intensive care unit; OHIR: Open-Heart Intraoperative Risk; OR: odds ratio; CI: confidence intervals.

TABLE 4: The OHIR score model for predicting a prolonged ICU stay.

\begin{tabular}{lcc}
\hline \multirow{2}{*}{ Risk factors } & \multicolumn{2}{c}{ Score } \\
& Present & Absent \\
\hline Age $(\geq 60$ years $)$ & 1 & 0 \\
P/F ratio $(\leq 200 \mathrm{mmHg})$ & 1 & 0 \\
Platelet count $\left(\leq 120,000 / \mathrm{mm}^{3}\right)$ & 1 & 0 \\
Inotrope/vasopressor requirement $(\geq 2$ drugs) & 2 & 0 \\
Serum potassium $(\leq 3.2 \mathrm{mEq} / \mathrm{L})$ & 1 & 0 \\
Atrial fibrillation $($ grading $\geq 2)$ & 1 & 0 \\
\hline OHIR score: Open-Heart Intraoperative Risk score; total score $=7$; score $\geq 3$ \\
suggests a prolonged stay in intensive care unit.
\end{tabular}

TABLE 5: The sensitivity, specificity, positive predictive value, and negative predictive value of OHIR score at cut-off point $\geq 3$ in this study.

\begin{tabular}{lcc}
\hline OHIR score & Value $(\%)$ & $95 \%$ CI \\
\hline Sensitivity & 72.3 & $60.2-82.6$ \\
Specificity & 76.5 & $66.8-84.1$ \\
Positive predictive value & 66.7 & $54.5-77.1$ \\
Negative predictive value & 81.2 & $71.7-88.2$ \\
Accuracy & 75 & - \\
Likelihood ratio & 3.09 & - \\
AUC for ROC of OHIR & 0.746 & $0.668-0.824$ \\
\hline
\end{tabular}

OHIR: Open-Heart Intraoperative Risk; CI: confidence intervals; AUC for ROC: the area under the receiver operating characteristic curve.

ICU stay increased with the OHIR score. Importantly, there was a significant gap between an OHIR score of 2 and 3 across our cut-off point of 42 hours.

During this study, there were neither deaths nor any serious complications, for example, coma, stroke, heart failure, acute renal failure, or respiratory failure.

\section{Discussion}

We developed the intraoperative predictive model (OHIR score) for a prolonged ICU stay after cardiac surgery with CPB. We first validated the modified SOFA score for its predictive capability in a pilot study. There are several prognostic
TABLE 6: Mean ICU stay for each OHIR score for the patients in this study.

\begin{tabular}{lccc}
\hline OHIR score & Number of patients & Mean (hours) & SD \\
\hline 0 & 28 & 28.62 & 17.37 \\
1 & 31 & 29.69 & 15.82 \\
2 & 37 & 38.50 & 22.88 \\
3 & 34 & 62.62 & 48.52 \\
4 & 23 & 71.37 & 61.07 \\
5 & 9 & 128.22 & 68.71 \\
6 & 4 & 51.75 & 21.87 \\
7 & 2 & 102.50 & 17.68
\end{tabular}

ICU: intensive care unit; OHIR: Open-Heart Intraoperative Risk; SD: standard deviation.

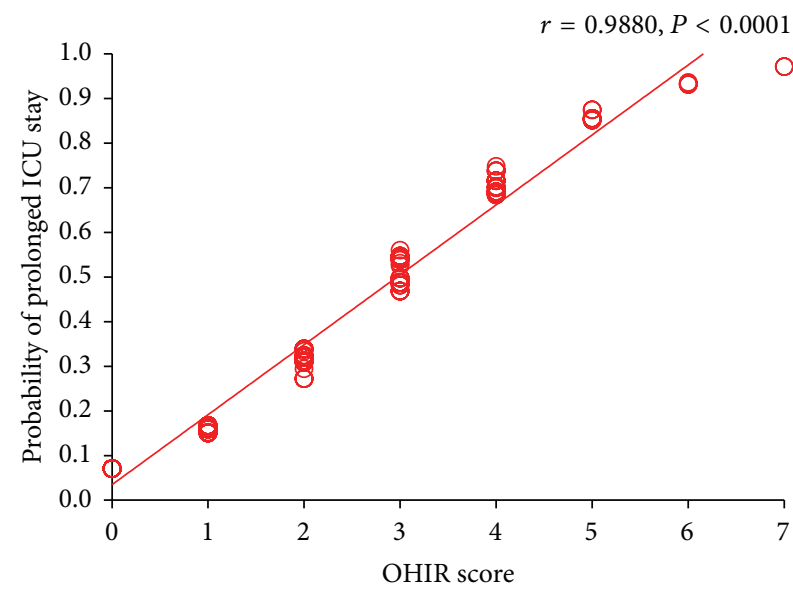

FIgURE 1: Correlation of Open-Heart Intraoperative Risk (OHIR) score and probability of a prolonged ICU stay, derived from the multivariate logistic regression model, presented as the Pearson correlation.

scoring models being used in ICU to assess the risks of systemic inflammatory response, sepsis and severe sepsis, and MODS/MOF of critically ill patients (e.g., APACHE, SOFA, and MOD) [20-23]. These have, however, never been used to evaluate risk of, or predict, a prolonged ICU stay among 
patients undergoing cardiac surgery with $\mathrm{CPB}$. If these scores can assess the severity of inflammation of patients in the ICU, they may be used in patients undergoing cardiac surgery to predict a prolonged ICU stay.

We chose the SOFA score because (a) it includes risk factors relevant to surgery and (b) it is practicable at bedside. We modified it by omitting the Glasgow coma score because the patients were unconscious under general anesthesia. The modified SOFA scores 3 hours after CPB insertion yielded the best prediction of a prolonged ICU stay. We chose all variables relevant at this juncture to develop the new model because this juncture is considered optimal for assessing prognosis since it is sufficiently early for the attending anesthesiologists to ameliorate the correctable risk factors so as to improve postoperative outcomes.

The modified SOFA score can accurately predict a prolonged ICU stay (with an AUC for ROC of 0.849). The Parsonnet and EuroSCORE, as well as the modified SOFA score, were initially developed to predict mortality but they also have the scope for predicting prolonged ICU stay $[5,24-26]$. The modified SOFA score is inappropriate for intraoperative use as it consists of too few intraoperative risk factors (only 4), but it seeded development of this new model.

The definition of a prolonged ICU stay varies among studies from $>24$ hours to $>3$ days [5]. For inferential purposes, we used statistic-based criteria, that is, length of stay in ICU longer than the median, which was 42 hours in our hospital.

Our multivariate logistic regression model consisted of 6 risk factors. Although the original model had both high discrimination and precision, the formula was complicated and difficult to calculate. We, therefore, modified it into a simple scoring model and called it the OHIR score, which contains the same risk factors but with a simplified formula, easy to calculate at bedside. Both models provided excellent linear correlation indicating that they could substitute each other and both showed very good discrimination vis-à-vis the external validation test.

This is the first model for intraoperative use, to predict the prognosis of the patients undergoing cardiac surgery. From the model, all except one risk factor (i.e., age $\geq 60$ years) are manageable, meaning that if these factors are improved in the patient, the OHIR score can be reduced and the probability of a prolonged ICU stay may be decreased.

Many predictive models have been developed for a prolonged ICU stay-for example, the Parsonnet, EuroSCORE, Tuman, TU, Pitkanen, Hujdkes, Christakis, Wong, Ivanov, Janssen, Abrahamyam, and Ghotkar models [5, 24, 25]. These are, however, for different populations with differences in definitions of outcomes and predictors. All of them, moreover, are preoperative evaluation tools, and none can be used as an intraoperative tool, unlike our model, which was developed by expanding the scope of the modified SOFA score. Our OHIR model consists of 6 risk factors, 5 of which are manipulatable. We suggest that it may be a useful intraoperative tool for (a) assessing prognosis, (b) providing the anesthesiologist with enough time to correct the risk factors, thereby (c) improving both anesthetic care and patient outcomes, and (d) lowering the costs of patient care.

4.1. Limitations. Although we planned to treat and discharge the patients according to the mentioned protocols, the time when the patients were transferred into or out of ICU may depend greatly on when the surgery was complete, availability of bed in ordinary ward, and the decision made in day or night shift. Although our sample size was small compared with other retrospective development models, this was a prospective study with an appropriate sample size calculation: our study, thus, demonstrates high discrimination and good precision. Despite the external validation of both the logistic regression and OHIR models in an independent group of patients, all of the patients remained in the same hospital. Further validation of the OHIR score in other institutions is, therefore, recommended.

\section{Conclusions}

We developed a simple intraoperative OHIR score for predicting prolonged ICU stay after cardiac surgery, starting with validation of the modified SOFA score. We used the risk factors from the modified SOFA score, plus those from an extensive literature review, to construct a predictive model using logistic regression analysis. The logistic regression model had good calibration and discrimination. This model was further simplified into the OHIR score. Both models had excellent correlation and were externally validated in the pilot study patients with good discrimination. The OHIR score is relatively simple and easy to calculate at bedside. It may be used as an intraoperative tool for improving the quality of anesthetic care.

\section{Conflict of Interests}

The authors declare that there is no conflict of interests regarding the publication of this paper.

\section{Acknowledgments}

The authors thank Associate Professor Dr. Bandit Thinkhamrop, Department of Biostatistics and Demography, Faculty of Public Health, Khon Kaen University, for advice on statistics and data analysis, Dr. Chanvit Leelayuwat, Department of Clinical Immunology, Faculty of Associated Medical Sciences, Khon Kaen University, Thailand, for his useful comments, Professor Dr. Yukifumi Nawa for guidance in paper preparation, and Mr. Bryan Roderick Hamman for assistance with the English language presentation. This study was funded by the Faculty of Medicine, Khon Kaen University, Khon Kaen, Thailand.

\section{References}

[1] H. R. Girn, S. Ahilathirunayagam, A. I. D. Mavor, and S. HomerVanniasinkam, "Basic science review: reperfusion syndrome: 
cellular mechanisms of microvascular dysfunction and potential therapeutic strategies," Vascular and Endovascular Surgery, vol. 41, no. 4, pp. 277-293, 2007.

[2] G. Orhan, M. Sargin, S. Senay et al., "Systemic and myocardial inflammation in traditional and off-pump cardiac surgery," Texas Heart Institute Journal, vol. 34, no. 2, pp. 160-165, 2007.

[3] J. G. Laffey, J. F. Boylan, and D. C. H. Cheng, "The systemic inflammatory response to cardiac surgery," Anesthesiology, vol. 97, no. 1, pp. 215-252, 2002.

[4] K. Werdan, H. Schmidt, H. Ebelt et al., "Impaired regulation of cardiac function in sepsis, SIRS, and MODS," Canadian Journal of Physiology and Pharmacology, vol. 87, no. 4, pp. 266-274, 2009.

[5] R. G. A. Ettema, L. M. Peelen, M. J. Schuurmans, A. P. Nierich, C. J. Kalkman, and K. G. M. Moons, "Prediction models for prolonged intensive care unit stay after cardiac surgery: systematic review and validation study," Circulation, vol.122, no. 7, pp. 682-689, 2010.

[6] E. Cholongitas, M. Senzolo, D. Patch, S. Shaw, C. Hui, and A. K. Burroughs, "Review article: scoring systems for assessing prognosis in critically ill adult cirrhotics," Alimentary Pharmacology and Therapeutics, vol. 24, no. 3, pp. 453-464, 2006.

[7] S. Senay, F. Toraman, H. Karabulut, and C. Alhan, "Is it the patient or the physician who cannot tolerate anemia? A prospective analysis in 1854 non-transfused coronary artery surgery patients," Perfusion, vol. 24, no. 6, pp. 373-380, 2009.

[8] K. Giakoumidakis, G. I. Baltopoulos, C. Charitos, E. Patelarou, P. Galanis, and H. Brokalaki, "Risk factors for prolonged stay in cardiac surgery intensive care units," Nursing in Critical Care, vol. 16, no. 5, pp. 243-251, 2011.

[9] J. de Cocker, N. Messaoudi, B. A. Stockman, L. L. Bossaert, and I. E. R. Rodrigus, "Preoperative prediction of intensive care unit stay following cardiac surgery," European Journal of Cardiothoracic Surgery, vol. 39, no. 1, pp. 60-67, 2011.

[10] G.-W. He, T. E. Acuff, W. H. Ryan, Y.-H. He, and M. J. Mack, "Influence of old age, gender, and internal mammary artery grafting on operative mortality and morbidity in coronary artery bypass grafting," American Journal of Geriatric Cardiology, vol. 5, no. 6, pp. 22-35, 1996.

[11] K. H. Polderman and A. R. J. Girbes, "Severe electrolyte disorders following cardiac surgery: a prospective controlled observational study," Critical Care, vol. 8, no. 6, pp. R459-R466, 2004.

[12] A. Shander, "Anemia in the critically ill," Critical Care Clinics, vol. 20, no. 2, pp. 159-178, 2004.

[13] F. Filsoufi, J. Jouan, J. Chilkwe et al., "Results and predictors of early and late outcome of coronary artery bypass graft surgery in patients with ejection fraction less than 20\%," Archives of Cardiovascular Diseases, vol. 101, no. 9, pp. 547-556, 2008.

[14] J. Wang, F. Xiao, J. Ren, Y. Li, and M.-L. Zhang, "Risk factors for mortality after coronary artery bypass grafting in patients with low left ventricular ejection fraction," Chinese Medical Journal, vol. 120, no. 4, pp. 317-322, 2007.

[15] S. Bramer, A. H. M. van Straten, M. A. Soliman Hamad, E. Berreklouw, E. J. Martens, and J. G. Maessen, "The impact of new-onset postoperative atrial fibrillation on mortality after coronary artery bypass grafting," Annals of Thoracic Surgery, vol. 90, no. 2, pp. 443-449, 2010.

[16] R. P. Villareal, R. Hariharan, B. C. Liu et al., "Postoperative atrial fibrillation and mortality after coronary artery bypass surgery," Journal of the American College of Cardiology, vol. 43, no. 5, pp. 742-748, 2004.
[17] J. C. Hart, "Maintaining hemodynamic stability and myocardial performance during Off-pump coronary bypass surgery," Annals of Thoracic Surgery, vol. 75, no. 2, pp. S740-S744, 2003.

[18] Q. Lei, L. Chen, Y. Zhang, N. Fang, W. Cheng, and L. Li, "Predictors of prolonged mechanical ventilation after aortic arch surgery with deep hypothermic circulatory arrest plus antegrade selective cerebral perfusion," Journal of Cardiothoracic and Vascular Anesthesia, vol. 23, no. 4, pp. 495-500, 2009.

[19] B. G. Tabachnick and L. S. Fidell, Using Multivariate Statistics, Pearson Allyn \& Bacon, Upper Saddle River, NJ, USA, 5th edition, 2007.

[20] S. Y. Hwang, J. H. Lee, Y. H. Lee et al., "Comparison of the Sequential Organ Failure Assessment, Acute Physiology and Chronic Health Evaluation II scoring system, and Trauma and Injury Severity Score method for predicting the outcomes of intensive care unit trauma patients," American Journal of Emergency Medicine, vol. 30, pp. 749-753, 2012.

[21] J. C. Marshall, D. J. Cook, N. V. Christou, G. R. Bernard, C. L. Sprung, and W. J. Sibbald, "Multiple organ dysfunction score: a reliable descriptor of a complex clinical outcome," Critical Care Medicine, vol. 23, no. 10, pp. 1638-1652, 1995.

[22] M. J. Breslow and O. Badawi, "Severity scoring in the critically ill-part 1-interpretation and accuracy of outcome prediction scoring systems," Chest, vol. 141, no. 1, pp. 245-252, 2012.

[23] M. J. Breslow and O. Badawi, "Severity scoring in the critically ill-part 2-maximizing value from outcome prediction scoring systems," Chest, vol. 141, no. 2, pp. 518-527, 2012.

[24] V. Parsonnet, D. Dean, and A. D. Bernstein, "A method of uniform stratification of risk for evaluating the results of surgery in acquired adult heart disease," Circulation, vol. 79, no. 6, pp. 3$12,1989$.

[25] S. A. M. Nashef, F. Roques, P. Michel, E. Gauducheau, S. Lemeshow, and R. Salamon, "European system for cardiac operative risk evaluation (EuroSCORE)," European Journal of Cardiothoracic Surgery, vol. 16, no. 1, pp. 9-13, 1999.

[26] S. A. M. Nashef, F. Roques, B. G. Hammill et al., "Validation of European System for Cardiac Operative Risk Evaluation (EuroSCORE) in North American cardiac surgery," European Journal of Cardiothoracic Surgery, vol. 22, no. 1, pp. 101-105, 2002. 


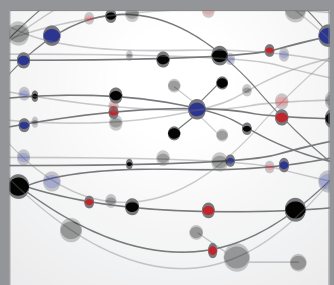

The Scientific World Journal
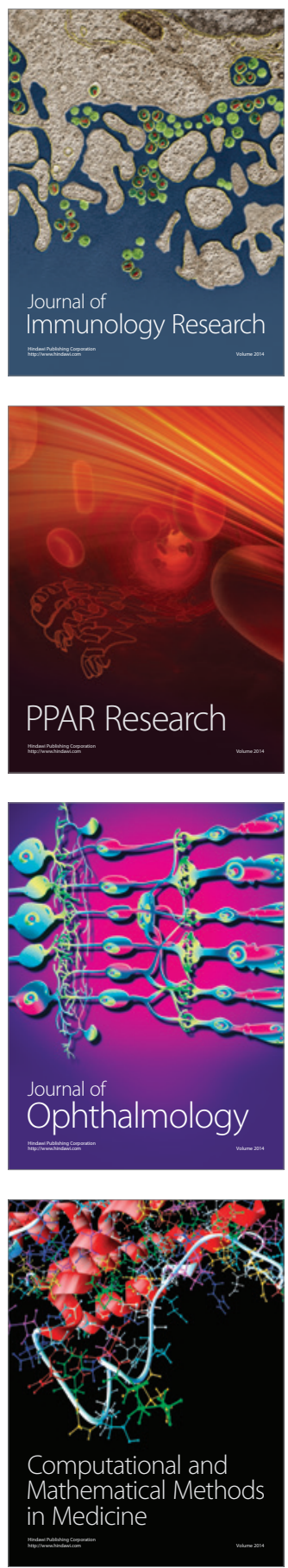

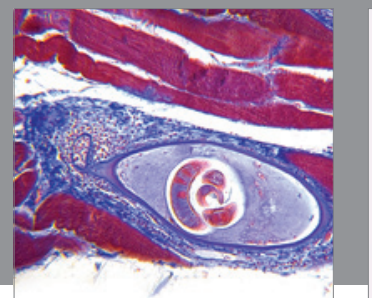

Gastroenterology

Research and Practice
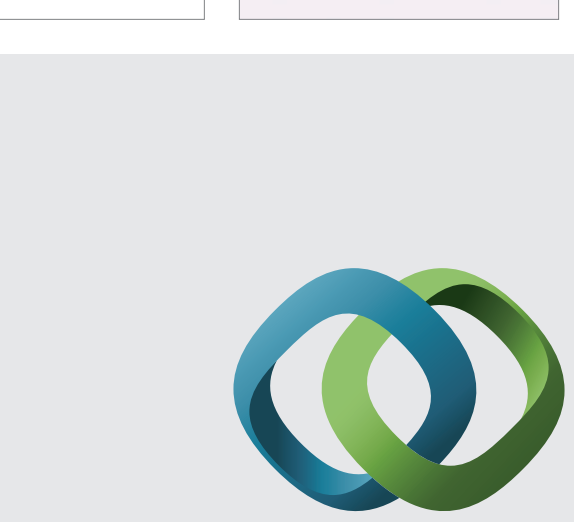

\section{Hindawi}

Submit your manuscripts at

http://www.hindawi.com
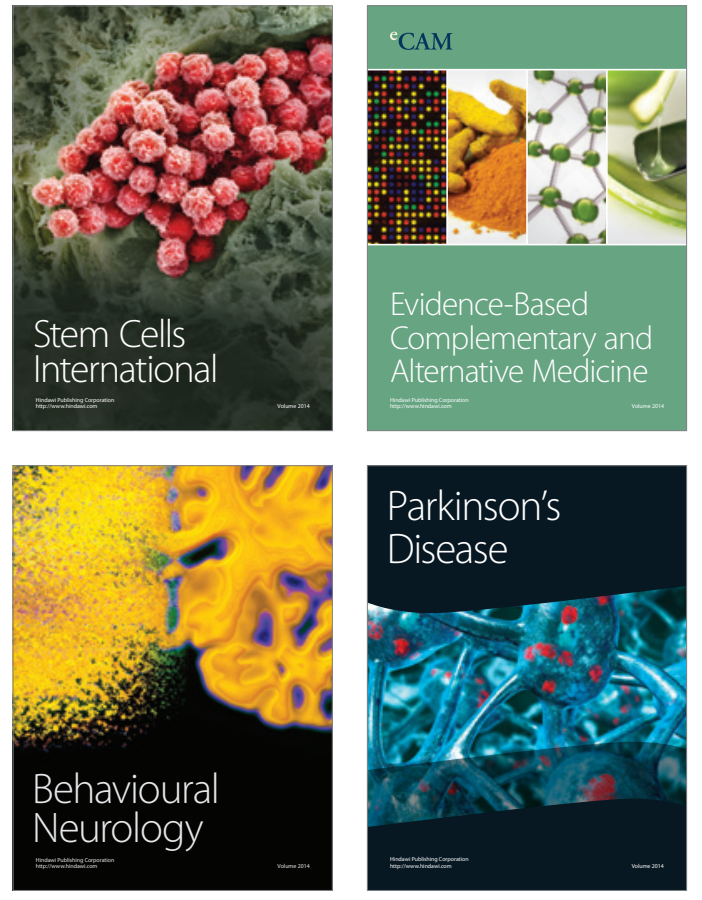
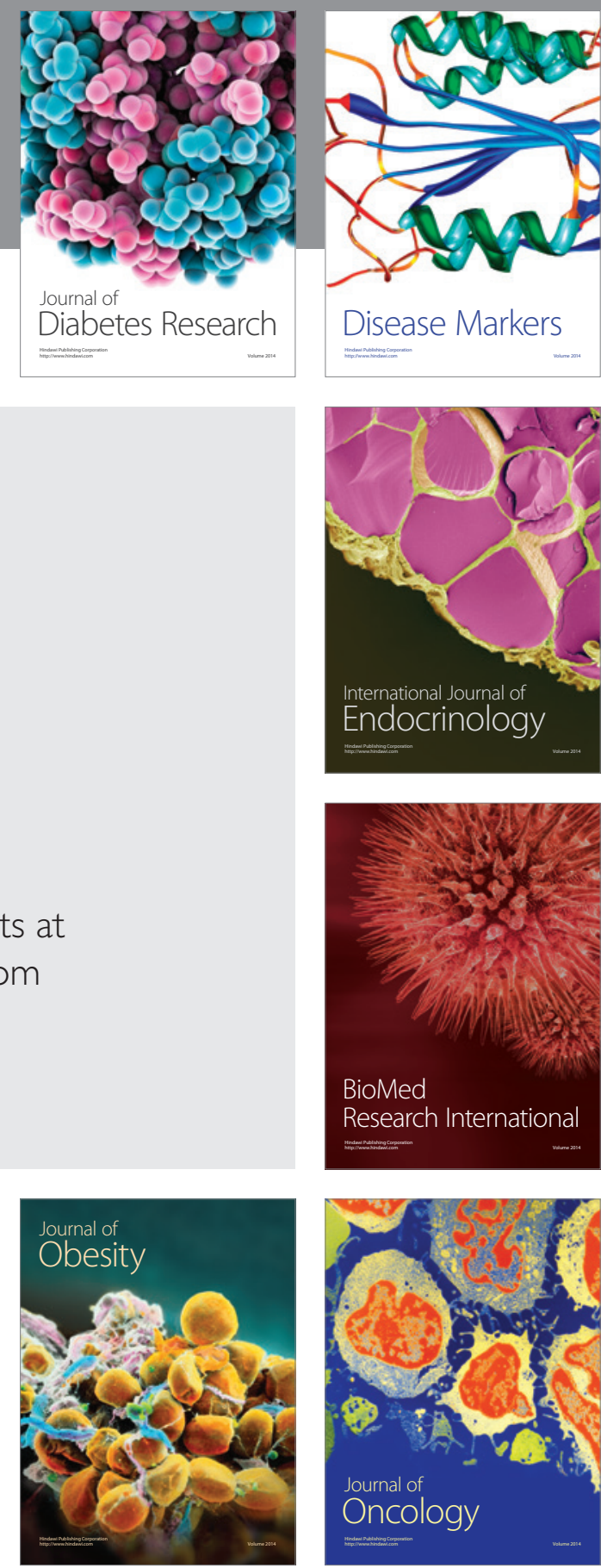

Disease Markers
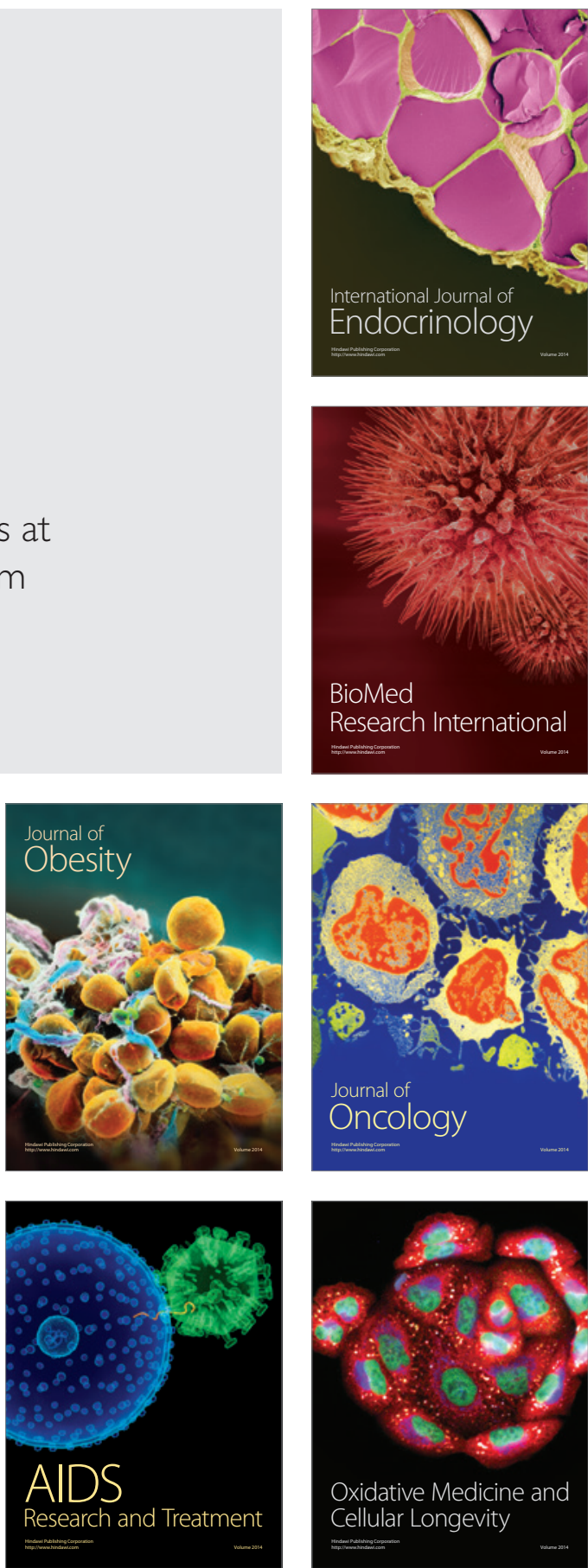